\title{
Tight bound on trace distance between a realistic device with partially indistinguishable bosons and the ideal Boson Sampling
}

\author{
V. S. Shchesnovich \\ Centro de Ciências Naturais e Humanas, Universidade Federal do ABC, Santo André, SP, 09210-170 Brazil
}

\begin{abstract}
We study the closeness of an experimental unitary bosonic network with only partially indistinguishable bosons in an arbitrary mixed input state, in particular an experimental realization of the Boson Sampling, to the ideal bosonic network, where the measure of closeness of two networks is the trace distance between the output probability distributions. An upper bound on the trace distance to the ideal bosonic network is proven and also a bound on the difference between probabilities of an output configuration. Moreover, the upper bound on the trace distance is tight, provided that a physically transparent distinguishability conjecture is true. For a small distinguishability error it is shown that a realistic device with $N$ bosons is at a constant trace distance to the ideal Boson Sampling under the $O\left(N^{-1}\right)$-scaling of the mismatch of internal states of bosons.

PACS numbers: $\quad$ 42.50.St, 03.67.Ac, 42.50.Ar
\end{abstract}

\section{INTRODUCTION}

The Boson Sampling (BS) of S. Aaronson and A. Arkhipov [1] can potentially become the first operating device with postclassical computational power, if scaled up to few dozens of bosons. It can be implemented with passive linear optical devices, single-photon sources, and non-adaptive detection of photons, clearly not enough for the universal quantum computation [24]. A complex interference of bosonic path amplitudes in a unitary linear network elevates the output probability distribution of the $\mathrm{BS}$ to the \#P class of computational complexity [5 7], asymptotically inaccessible for a classical computer even to verify the result. The key point in optical implementation of the BS is the quantum indistinguishability of single photons, resulting in the Hong-Ou-Mandel dip [8] (see also Ref. [9]). Several labs have performed the proof of principle experiments on small networks with few single photons 10 14. Various error models have been analyzed [15-18]. It is now clear that an experimental device with postclassical computational power is feasible, if setup errors are properly tuned [19]. An experimental implementation can now be chosen between various setups [20 22]. Though no unconditional verification method of an operating device is known, there are results in this direction [23] with an experimental confirmation [24, 25]. Moreover, there is a conditional certification protocol based on many-body interference [26]. Recently, it was shown that the Franck-Condon transition probabilities in molecular vibronic spectra can be simulated on the BS with a modified input [27].

In the present work we consider how to measure the closeness between a realistic BS device, implemented with only partially indistinguishable bosons, and the ideal BS. We use the trace distance between the output probability distributions of a realistic device and the ideal BS as the measure of their closeness and study how to estimate the trace distance using the information derivable from experiment. We give an upper bound on the trace distance, and show that under a highly plausible physically clear conjecture the derived bound is tight. We employ a recently developed partial indistinguishability theory [28] in our derivation of the upper bound on the trace distance. In fact, we derive the main results in a more general form, so that they can be applied to measure the closeness of an arbitrary experimental bosonic network, with an arbitrary (mixed) input state of bosons, to the ideal bosonic one with the same distribution of particles over the input modes. Thus one can use the upper bound derived in the present work to quantify the "bosonness" of an experimental network. Moreover, the upper bound on the trace distance between a realistic and the ideal bosonic networks involves the probability of the maximally bunched output (when all particles are in the same output mode), which can be estimated from the experimental data.

Here it should be noted that the purity measure of partial indistinguishability, proposed in Ref. [28], which quantifies the quality of a quantum interference in an unitary linear network (for instance, it reduces to the Mandel's parameter 29] for two bosons) is not suitable, in general, for attesting how close is a realistic network to the ideal bosonic network (i.e., with the output amplitudes proportional to the matrix permanents of submatrices of the network matrix). This is due to the fact that both species of identical particles, bosons and fermions, can behave in an interference experiment as the other species, if the particles are in an appropriate (entangled) input state (one can even simulate the BS with fermions) [30]. For instance, the output probability distribution of a bosonic network with bosons maximizing the purity measure of Ref. 28] (thus being completely indistinguishable particles) can be identically equivalent to that of the ideal fermionic one, if the input state is anti-symmetric with respect to permutation of internal states of particles. In the latter case the bosonic network is still a perfectly quantum network (that is why it has the maximal purity), but equivalent to the ideal fermionic network. Only in the case when the input state of bosons is just 
a small perturbation of a state symmetric with respect to internal states of particles, the purity measure can be used to quantify how close such a network is to the ideal bosonic one. In general case, to measure the closeness to the ideal bosonic network a new specific measure is required, which must reflect how the internal state of bosons (the projection of the input state of particles on the Hilbert space of the degrees of freedom not affected by a network) is close to a nearest symmetric state, since only such a symmetric input state results in the ideal bosonic behavior [28].

Since there is a continuous family of input states for every probability distribution realizable at output of an unitary network [28], it is more convenient to work with the partial indistinguishability matrix ( $J$-matrix) of Refs. [18, 28], which is the unique image of all input states of bosons resulting in the same output probability distribution in a unitary network. A linear space $\mathcal{V}_{N}$ of dimension $N$ ! for $N$ particles can be associated with $J$-matrices, where vectors and matrices are labeled by elements (permutations) of the symmetric group $\mathcal{S}_{N}$. A crucial point is that a boson counting measurement at network output is formally a measurement also in the auxiliary linear space $\mathcal{V}_{N}$. Thanks to this fact, by application of the standard methods we derive a uniform (over all networks) upper bound on the trace distance of an output probability distribution to that of the ideal bosonic network, which supersedes the probabilistic bound of Ref. [18]. Under a physically transparent distinguishability conjecture, there is also a lower bound on the trace distance, which has the same scaling in the total number of bosons as the upper bound, thus making the latter tight. The distinguishability conjecture is a restricted from of a highly plausible property that different $J$-matrices result in distinguishable probability distributions, when the latter are compared over all possible networks.

In the case of bosons in pure spectral states, our upper bound on the trace distance to the ideal bosonic network is proportional to a recently proposed permanental measure [31]. However, our bound is tighter by $1 / N$ ! (the proportionality factor) for $N$ bosons.

The rest of the text is organized as follows. In an auxiliary section $\amalg \mathrm{A}$ we give a brief account of the results of Ref. 28] used below. In section \B we prove Theorem 1 (the main result), giving the upper bound on the trace distance to the output probability distribution of the ideal bosonic network. Some mathematical details of the derivation are placed in appendix $\mathrm{A}$. In section IIC we consider a special case of bosons in pure internal states. In section IID we derive a bound on the difference between probabilities of an output configuration, Theorem 2 (with some mathematical details placed in appendix (B). In section III we study the limit of a small distinguishability error and give a scaling law of the mismatch of internal states of bosons with the total number of particles for a constant trace distance to the ideal bosonic network. In section [V] we consider a lower bound on the trace distance and argue that under a highly plausible distinguishability conjecture our upper bound is tight. In section $\nabla$ we give concluding remarks.

\section{MAIN RESULTS}

\section{A. Output probability formula for a general case of partially indistinguishable bosons}

Let us first recall the necessary details of the partial indistinguishability theory in a unitary linear bosonic network (see for more details Ref. [28]).

Consider a linear unitary $M$-mode network with $N$ identical bosons at its input, such that each input mode $k=1, \ldots, M$ receives a certain number of particles $n_{k} \geq 0$. The single-particle degrees of freedom are divided into two parts. One part is composed of modes operated on by a linear unitary network. The other part consists of internal states unaffected by the network (whose Hilbert space will be denoted by $\mathcal{H}$ ). A network is given by a unitary matrix $U$, which leaves the Hilbert space of internal states invariant, i.e., acting as follows

$$
a_{k, j}^{\dagger}=\sum_{l=1}^{M} U_{k l} b_{l, j}^{\dagger},
$$

where $a_{k, j}^{\dagger} / b_{k, j}^{\dagger}$ creates a boson in an input/output mode $k$ and an internal state $\left|\phi_{j}\right\rangle$ (the set of states $\left\{\left|\phi_{1}\right\rangle, \ldots,\left|\phi_{j}\right\rangle, \ldots\right\}$ is a basis of $\left.\mathcal{H}\right)$. A general input state of configuration $\vec{n}=\left(n_{1}, \ldots, n_{M}\right)$ (we will use such vector notations below) reads $\rho(\vec{n})=\sum_{i} q_{i}\left|\Psi_{i}(\vec{n})\right\rangle\left\langle\Psi_{i}(\vec{n})\right|$ with $q_{i} \geq 0, \sum_{i} q_{i}=1$, and

$$
\left|\Psi_{i}(\vec{n})\right\rangle=\frac{1}{\sqrt{\mu(\vec{n})}} \sum_{\vec{j}} C_{\vec{j}}^{(i)} \prod_{\alpha=1}^{N} a_{k_{\alpha}, j_{\alpha}}^{\dagger}|0\rangle,
$$

where $\mu(\vec{n}) \equiv \prod_{k=1}^{M} n_{k}$ !, $k_{1}, \ldots, k_{N}$ are (generally repeated) input modes, and $|0\rangle$ is the vacuum state.

Due to permutation symmetry of boson creation operators, the expansion coefficients $C_{\vec{j}}^{(i)}$ can be chosen to satisfy permutation symmetry with respect to a Young subgroup of the symmetric group $\mathcal{S}_{N}$, consisting of permutations $\pi$ of internal states of bosons in each input mode between themselves, $\pi \in \mathcal{S}_{n_{1}} \otimes \ldots \otimes \mathcal{S}_{n_{M}} \equiv \mathcal{S}_{\vec{n}}$, where $\mathcal{S}_{n}$ is the symmetric group of $n$ objects. Such coefficients are normalized by $\sum_{\vec{j}}\left|C_{\vec{j}}^{(i)}\right|^{2}=1$.

For a given unitary network, probability of detection of an output configuration $\vec{m}=\left(m_{1}, \ldots, m_{M}\right),|\vec{m}|=N$ with $|\vec{m}| \equiv m_{1}+\ldots+m_{M}$, depends on the degree of partial indistinguishably of bosons, defined by their internal states. Moreover, experimental particle detection is imperfect, prone to particle losses and dark counts, besides introducing an effective filtering of the internal states due to non-ideal sensitivity of detectors. We assume that such effects are small and consider the postselected case of all bosons being detected at the output of a network. There 
still remains to consider the effect of non-ideal sensitivity of detectors (e.g., of limited spectral width in the case of photons). Here we adopt the simplest model of identical detectors. For identical detectors the input state alone determines the bound on the postselected probability distribution at output of a unitary network (since in this case the same $J$-matrix, defined below, is assigned to any output configuration of bosons).

The particle counting measurement is therefore described by the following positive operator-valued measure $\{\Pi(\vec{m}),|\vec{m}|=N\}$, where each element reads (see the derivation for photons in appendix A of Ref. [18])

$$
\Pi(\vec{m})=\frac{1}{\mu(\vec{m})} \sum_{\vec{j}}\left[\prod_{\alpha=1}^{N} \Gamma_{j_{\alpha}}\right] \prod_{\alpha=1}^{N} b_{l_{\alpha}, j_{\alpha}}^{\dagger}|0\rangle\langle 0| \prod_{\alpha=1}^{N} b_{l_{\alpha}, j_{\alpha}},
$$

here $\vec{l}=\left(l_{1}, \ldots, l_{N}\right)$ is a set of output modes, generally repeated, corresponding to mode occupations $\vec{m}$, $0 \leq \Gamma_{j} \leq 1$ is sensitivity of a detector to an internal state $\left|\phi_{j}\right\rangle$. In the case of non-ideal photon counting detectors, the summation over discrete internal states is replaced by an integral over spectral states [28]. Probability of detection of an output configuration $\vec{m}$ reads

$$
\hat{p}(\vec{m} \mid \vec{n})=\operatorname{Tr}\{\Pi(\vec{m}) \rho(\vec{n})\} .
$$

Inserting Eqs. (2) and (3) into Eq. (4) we obtain output probability as a quadratic form, which can be expressed as a double sum over $\mathcal{S}_{N}$ [18, 28]

$$
\hat{p}(\vec{m} \mid \vec{n})=\frac{1}{\mu(\vec{m}) \mu(\vec{n})} \sum_{\sigma_{1}, \sigma_{2}} J_{\sigma_{1}, \sigma_{2}} \prod_{\alpha=1}^{N} U_{k_{\sigma_{1}(\alpha)}, l_{\alpha}}^{*} U_{k_{\sigma_{2}(\alpha)}, l_{\alpha}} .
$$

The partial indistinguishability matrix $J$ indexed by two permutations $\sigma_{1}, \sigma_{2} \in \mathcal{S}_{N}$ (which has only up to $N$ ! different elements for identical detectors) is given by a trace

$$
J_{\sigma_{1}, \sigma_{2}}=\operatorname{Tr}\left(\hat{\Gamma}^{\otimes N} \rho^{(i n t)} P_{\sigma_{1} \sigma_{2}^{-1}}\right),
$$

where $\hat{\Gamma} \equiv \sum_{j} \Gamma_{j}\left|\phi_{j}\right\rangle\left\langle\phi_{j}\right|, P_{\sigma}$ is a representation of $\sigma \in$ $\mathcal{S}_{N}$ in $\mathcal{H}^{\otimes N}$, defined as

$P_{\sigma}\left|\phi_{j_{1}}\right\rangle \otimes \ldots \otimes\left|\phi_{j_{N}}\right\rangle=\left|\phi_{j_{\sigma^{-1}(1)}}\right\rangle \otimes \ldots \otimes\left|\phi_{j_{\sigma^{-1}(N)}}\right\rangle$,

and $\rho^{(i n t)}$ is an internal state of bosons corresponding to an input state of Eq. (2), i.e., $\rho^{(i n t)}=$ $\sum_{i} q_{i}\left|\Psi_{i}^{(i n t)}\right\rangle\left\langle\Psi_{i}^{(i n t)}\right|$ with

$$
\left|\Psi_{i}^{(i n t)}\right\rangle \equiv \sum_{\vec{j}} C_{\vec{j}}^{(i)} \prod_{\alpha=1}^{N}\left|\phi_{j_{\alpha}}\right\rangle .
$$

The ideal bosonic behavior in a unitary linear network occurs for $J_{\sigma_{1}, \sigma_{2}}^{(i d)}=1$ for all $\sigma_{1,2}$. It is known that this case realizes if and only if the internal state of bosons satisfies $S_{N} \rho^{(i n t)} S_{N}=\rho^{(i n t)}$ [28], where $S_{N}=\frac{1}{N !} \sum_{\sigma} P_{\sigma}$ is the projector on the symmetric subspace of $\mathcal{H}^{\otimes N}$. In this case Eq. (5D) contains the absolute value squared of the matrix permanent of a matrix $U[\vec{n} \mid \vec{m}]$, built from a network matrix $U$ by taking rows with multiplicities $\vec{n}$ and columns with multiplicities $\vec{m}$. For ideal detectors $\Gamma_{l, j}=1$ and

$$
\hat{p}^{(i d)}(\vec{m} \mid \vec{n})=\frac{|\operatorname{per}(U[\vec{n} \mid \vec{m}])|^{2}}{\mu(\vec{m}) \mu(\vec{n})} .
$$

In particular, the BS setup corresponds to an input with $n_{k} \leq 1$ in the dilute limit $M \gg N^{2}$. In this case probability of a bunched output (i.e., with some $m_{k} \geq 2$ ) vanishes as $O\left(N^{2} / M\right)$ and in the asymptotic limit $N \rightarrow \infty$ the output probability distribution cannot be simulated on a classical computer [1, 5, 7]. Recently it was argued that for $M \geq N^{2}$ the many-body correlations of indistinguishable particles may not vanish in the thermodynamic limit 32]. On the other hand, it is also known that for $M \ll N$ the output probability distribution can be approximated to a small multiplicative error by a semiclassical approach 33].

For non-ideal detectors, $\Gamma_{j} \neq 1$, output probabilities $\hat{p}(\vec{m} \mid \vec{n})$ of Eq. (5) do not sum to 1 . To compare with the ideal network Eq. (9), we consider the postselected probabilities, when $N$ input particles are detected at a network output. The postselected output probabilities are given by division of those of Eq. (4) by the detection probability $p_{d}=\sum_{\vec{m}} \hat{p}(\vec{m} \mid \vec{n})$. We have (see also Ref. [28])

$$
p_{d}=\frac{\operatorname{tr} J}{N !}=\operatorname{Tr}\left(\hat{\Gamma}^{\otimes N} \rho^{(i n t)}\right),
$$

where $\operatorname{tr}(A) \equiv \sum_{\sigma} A_{\sigma, \sigma}$ is the trace in the linear space $\mathcal{V}_{N}$

Finally, there is a continuous family of internal states of bosons resulting in the same probability distribution at output of a unitary linear network 28]. However, it is highly plausible that no two different $J$-matrices give coinciding output probability distributions over all unitary networks 37. Our main results, formulated below, do not change if the above property is not true in general (see also section IV).

\section{B. Upper bound on the trace distance to the ideal bosonic network}

We will work in the linear space $\mathcal{V}_{N}$, where $J$-matrix lives (for convenience, Dirac's notations will be used for vectors in $\mathcal{V}_{N}$, e.g., a vector of the natural basis, in which $J$-matrix is given by Eq. (6), will be denoted by $|\sigma\rangle$, $\left.\sigma \in \mathcal{S}_{N}\right)$. We consider how close is the postselected output probability distribution of a realistic bosonic network to the ideal bosonic network. The postselected output probability distribution will be denoted by $\mathbf{p}$, whereas a particular output probability by $p(\vec{m})$ (dropping the input configuration argument $\vec{n}$ ). The output probability distribution of the ideal bosonic network is denoted by 
$\mathbf{p}^{(i d)}$, a particular output probability $p^{(i d)}(\vec{m})$ is given by Eq. (9). Our goal is to estimate the trace distance

$$
D\left(\mathbf{p}^{(i d)}, \mathbf{p}\right)=\frac{1}{2} \sum_{\vec{m}}\left|p^{(i d)}(\vec{m})-p(\vec{m})\right| .
$$

The trace distance is a 1-norm based measure of closeness between two probability distributions (all norm based measures in a finite-dimensional linear space are equivalent). It is used in discussion of the computational complexity of a non-ideal BS (for details, see Ref. [1]). The following result is found.

Theorem 1.- The trace distance Eq. (11) satisfies

$$
D\left(\mathbf{p}^{(i d)}, \mathbf{p}\right) \leq 1-p_{s}
$$

with $p_{s}$ defined as follows

$$
p_{s}=\frac{1}{p_{d}} \operatorname{Tr}\left\{\hat{\Gamma}^{\otimes N} S_{N} \rho^{(i n t)} S_{N}\right\}=\frac{1}{p_{d}} \operatorname{Tr}\left\{\hat{\Gamma}^{\otimes N} \rho^{(i n t)} S_{N}\right\}
$$

(the second form is due to mutual commutation of $S_{N}$ and $\hat{\Gamma}^{\otimes N}$ and that $S_{N}$ is a projector).

Note that $p_{s}$ is the postselected (for non-ideal detectors) probability of a symmetric internal state of bosons at a network input. For a symmetric input state $S_{N} \rho^{(i n t)}=\rho^{(i n t)}$ we get $p_{s}=1$. In section IC below it is shown that the only parameter in the upper bound, $p_{s}$, can be estimated from the experimental data, i.e., even when no knowledge about the input state of particles is available.

Proof of Theorem 1.- The bound of Eqs. (12)-(13) easily follows from the following observation. Boson counting measurement at a network output can be thought of as a "measurement" also in the auxiliary linear space $\mathcal{V}_{N}$. Indeed, let us consider the trace-normalized $J$-matrix defined as follows (see Eq. (10))

$$
\mathcal{J}_{\sigma_{1}, \sigma_{2}} \equiv \frac{J_{\sigma_{1}, \sigma_{2}}}{N ! p_{d}}=\frac{1}{N ! p_{d}} \operatorname{Tr}\left\{\hat{\Gamma}^{\otimes N} \rho^{(i n t)} P_{\sigma_{1} \sigma_{2}^{-1}}\right\}
$$

This $\mathcal{J}$-matrix has all the properties of a density matrix in the linear space $\mathcal{V}_{N}$, whereas the following vectors $\left|Z_{\vec{l}}\right\rangle \in \mathcal{V}_{N}$ (here $\vec{l} \equiv\left(l_{1}, \ldots, l_{N}\right)$ the output mode indices), in the standard basis defined by

$$
\left\langle\sigma \mid Z_{\vec{l}}\right\rangle \equiv \frac{1}{\sqrt{\mu(\vec{n})}} \prod_{\alpha=1}^{N} U_{k_{\sigma(\alpha)}, l_{\alpha}}
$$

define a positive operator-valued measure in $\mathcal{V}_{N}$ :

$$
\sum_{\vec{l}}\left|Z_{\vec{l}}\right\rangle\left\langle Z_{\vec{l}}\right|=\mathcal{P}
$$

with the projector $\mathcal{P}$ given as

$$
\mathcal{P}_{\sigma, \sigma^{\prime}}=\frac{1}{\mu(\vec{n})} \sum_{\pi \in \mathcal{S}_{\vec{n}}} \delta_{\sigma^{\prime}, \pi \sigma}, \quad \operatorname{tr}(\mathcal{P})=\frac{N !}{\mu(\vec{n})}
$$

(see appendix $\mathrm{A}$ for more details). The projector $\mathcal{P}$ acts as the identity operator on any $\mathcal{J}$-matrix: $\mathcal{P} \mathcal{J}=\mathcal{J} \mathcal{P}=$ $\mathcal{J}$, due to the symmetry $\mathcal{J}_{\pi \sigma_{1}, \sigma_{2}}=\mathcal{J}_{\sigma_{1}, \pi \sigma_{2}}=\mathcal{J}_{\sigma_{1}, \sigma_{2}}$ for all $\pi$ from the Young subgroup $\mathcal{S}_{\vec{n}}=\mathcal{S}_{n_{1}} \otimes \ldots \otimes$ $\mathcal{S}_{n_{M}}$, as discussed in section IA The postselected output probability assumes a "measurement" form in the linear space $\mathcal{V}_{N}$

$$
p(\vec{m})=\sum_{m(\vec{l})=\vec{m}}\left\langle Z_{\vec{l}}|\mathcal{J}| Z_{\vec{l}}\right\rangle,
$$

where the summation is over all output mode indices $\vec{l}=$ $\left(l_{1}, \ldots, l_{N}\right)$ corresponding to an output configuration $\vec{m}$ (in total $N ! / \mu(\vec{m})$ terms).

The rest is an elementary algebra. The independence of the sum $\sum_{\sigma_{1}} \mathcal{J}_{\sigma, \sigma_{1}}$ from $\sigma$ (recall that $\mathcal{J}_{\sigma_{2}, \sigma_{1}}$ depends on the relative permutation $\sigma_{1} \sigma_{2}^{-1}$ only) means that such a $\mathcal{J}$-matrix has an eigenstate $|s\rangle$, whose expansion in the standard basis reads $\langle\sigma \mid s\rangle=1 / \sqrt{N}$ !, for any permutation $\sigma$. The corresponding eigenvalue is precisely the postselected probability of a symmetric input state $p_{s}$. Indeed, from Eq. (14) by comparing with Eq. (13) we get

$$
\langle s|\mathcal{J}| s\rangle=\frac{1}{N !} \sum_{\sigma_{1}, \sigma_{2}} \mathcal{J}_{\sigma_{1}, \sigma_{2}}=\sum_{\sigma} \mathcal{J}_{\sigma, I}=p_{s}
$$

where $I$ is the identity permutation. Therefore, $\mathcal{J}$-matrix as a convex combination of two matrices

$$
\mathcal{J}=p_{s}|s\rangle\left\langle s\left|+\left(1-p_{s}\right) \mathcal{J}^{(\perp)}, \quad \mathcal{J}^{(\perp)}\right| s\right\rangle=0 .
$$

Using Eq. (20) to rewrite the trace distance (11) as follows $D\left(\mathbf{p}^{(i d)}, \mathbf{p}\right)=\left(1-p_{s}\right) D\left(\mathbf{p}^{(i d)}, \mathbf{p}^{(\perp)}\right)$ (with $\mathbf{p}^{(\perp)}$ corresponding to $\left.\mathcal{J}^{(\perp)}\right)$, noting that the trace distance is bounded by 1, we get the upper bound of Eq. (12). Q.E.D.

Finally, it is notable that the upper bound Eq. (12) coincides with the trace distance between a given $\mathcal{J}$-matrix and the pure $\mathcal{J}^{(i d)}$-matrix of the ideal case $\mathcal{J}^{(i d)}=|s\rangle\langle s|$. We have 38]

$$
\mathcal{D}(|s\rangle\langle s|, \mathcal{J}) \equiv \frac{1}{2} \operatorname{tr}\{|| s\rangle\langle s|-\mathcal{J}|\}=1-p_{s},
$$

where we have used Eq. (20).

\section{Upper bound on the trace-distance for pure internal states of bosons}

It turns out that, for bosons in pure internal states, the probability $p_{s}$ defined in Eq. (13) is intimately related to the permanental measure of Ref. 31. Indeed, let $N$ bosons have pure internal states $\left|\varphi_{\alpha}\right\rangle, \alpha=1, \ldots, N$. We have from Eq. (6)

$$
J_{\sigma_{1}, \sigma_{2}}=\prod_{\alpha=1}^{N}\left\langle\varphi_{\sigma_{1}(\alpha)}|\hat{\Gamma}| \varphi_{\sigma_{2}(\alpha)}\right\rangle=\prod_{\alpha=1}^{N}\left\langle\varphi_{\alpha}|\hat{\Gamma}| \varphi_{\sigma_{2} \sigma_{1}^{-1}(\alpha)}\right\rangle
$$


thus

$$
p_{d}=\prod_{\alpha=1}^{N}\left\langle\varphi_{\alpha}|\hat{\Gamma}| \varphi_{\alpha}\right\rangle
$$

and

$$
p_{s}=\frac{1}{p_{d} N !} \sum_{\sigma} \prod_{\alpha=1}^{N}\left\langle\varphi_{\alpha}|\hat{\Gamma}| \varphi_{\sigma(\alpha)}\right\rangle=\frac{\operatorname{per}(G)}{N !},
$$

where the Gram matrix built from the internal states reads $G_{\alpha \beta}=\left\langle\widetilde{\varphi}_{\alpha}|\hat{\Gamma}| \widetilde{\varphi}_{\beta}\right\rangle$ with $\left|\widetilde{\varphi}_{\alpha}\right\rangle=\left|\varphi_{\alpha}\right\rangle /\left(\left\langle\varphi_{\alpha}|\hat{\Gamma}| \varphi_{\alpha}\right\rangle\right)^{\frac{1}{2}}$.

However, our bound given by Eq. (12) is tighter by $1 / N$ ! than the previous bound, $N !-\operatorname{per}(G)$, proposed in Ref. 31].

\section{Bound on the variation of an output probability from that of the ideal network}

Let us recall that the case of classically indistinguishable particles corresponds to $\mathcal{J}=\frac{\mu(\vec{n})}{N !} \mathcal{P}$ (the maximally mixed $\mathcal{J}$-matrix as allowed by the symmetry $\mathcal{P} \mathcal{J}=$ $\mathcal{J P}=\mathcal{J}$, see also Ref. [28]). The classical probability of an output configuration $\vec{m}$ reads

$$
p^{(c l)}(\vec{m})=\frac{\operatorname{per}\left(|U|^{2}[\vec{n} \mid \vec{m}]\right)}{\mu(\vec{m})},
$$

where $|U|^{2}[\vec{n} \mid \vec{m}]$ is a matrix constructed from elements $\left|U_{k l}\right|^{2}$ by taking rows with multiplicities $\vec{n}$ and columns with multiplicities $\vec{m}$. We are interested in how close is the postselected probability of an output configuration $\vec{m}$ in a realistic network to that of the ideal bosonic network. The following result is found.

Theorem 2.- The difference between the postselected probability of an output configuration $\vec{m}$ in a realistic bosonic network and that in the ideal bosonic one satisfies

$$
\left|p^{(i d)}(\vec{m})-p(\vec{m})\right| \leq\left(1-p_{s}\right) \frac{N !}{\mu(\vec{n})} p^{(c l)}(\vec{m})
$$

where $p_{s}$ is given by Eq. (13).

Proof of Theorem 2.- The bound (26) follows from Eqs. (18)-(20) and the following inequality

$$
\left|\sum_{m(\vec{l})=\vec{m}}\left\langle Z_{\vec{l}}|\mathcal{A}| Z_{\vec{l}}\right\rangle\right| \leq \sum_{m(\vec{l})=\vec{m}}\left\langle Z_{\vec{l}} \mid Z_{\vec{l}}\right\rangle=\frac{N !}{\mu(\vec{n})} p^{(c l)}(\vec{m}),
$$

valid for any Hermitian matrix $\mathcal{A}$ whose eigenvalues lie in the interval $[-1,1]$. Setting $\Delta \mathcal{J}=|s\rangle\langle s|-\mathcal{J}^{(\perp)}$, noticing that its eigenvalues lie in the interval $[-1,1]$, using Eq. (27), (18), and (20) we get

$$
\begin{aligned}
\left|p^{(i d)}(\vec{m})-p(\vec{m})\right| & =\left(1-p_{s}\right)\left|\sum_{m(\vec{l})=\vec{m}}\left\langle Z_{\vec{l}}|\Delta \mathcal{J}| Z_{\vec{l}}\right\rangle\right| \\
& \leq\left(1-p_{s}\right) \frac{N !}{\mu(\vec{n})} p^{(c l)}(\vec{m})
\end{aligned}
$$

i.e., the bound of Eq. (26). Q.E.D.

For bosons in pure internal states, as in section IC, Eq. (26) contains two factors: (i) the complementary of the probability of the ideal bosonic behavior $1-p_{s}=$ $1-\operatorname{per}(G) / N$ ! (see Eq. (24)) and (ii) the upper bound of Eq. (27) on output probability $p(\vec{m})$. Recently, a bound similar to that of Eq. (26) was proved for a real positive $G$-matrix of Eq. (24) and then numerically validated for the general case of bosons in pure states 31. In this respect, Theorem 2, besides being an extension to general mixed inputs, gives a generally tighter bound than that of Ref. [31], due to $\mu(\vec{n})$ in the denominator.

Finally, as was previously noted in Ref. [31], the probability $p_{s}$ can be read off from the experiment. Indeed, $p_{s}$ appears in the output probability (postselected for nonideal detectors) of a maximally bunched output configuration (see Refs. $\left[14,[34]\right.$ ), say $m_{l}^{(B)}=N$. In this case

$$
p^{(i d)}\left(\vec{m}^{(B)}\right)=\frac{N !}{\mu(\vec{n})} \prod_{\alpha=1}^{N}\left|U_{k_{\alpha}, l}\right|^{2}=\frac{N !}{\mu(\vec{n})} p^{(c l)}\left(\vec{m}^{(B)}\right)
$$

and we obtain (details in Appendix B)

$$
p\left(\vec{m}^{(B)}\right)=p_{s} \frac{N !}{\mu(\vec{n})} p^{(c l)}\left(\vec{m}^{(B)}\right),
$$

i.e., the same expression as in Ref. [31], extended here to arbitrary (mixed) internal states of bosons.

\section{SMALL ERRORS: SCALING OF THE DISTINGUISHABILITY ERROR WITH $N$}

Let us consider a practically relevant case of small errors, which allows for an analytic solution. We assume that detectors are nearly ideal $\delta \hat{\Gamma} \equiv 1-\hat{\Gamma} \ll 1$ and that bosons, originated from independent sources, have internal states which are approximately the same pure state, i.e., $\rho^{(\text {int })}=\prod_{\alpha=1}^{N}{ }^{\otimes} \rho_{\alpha}$, with $\rho_{\alpha}=|\phi\rangle\langle\phi|-\delta \rho_{\alpha}$ (note that $\operatorname{Tr}\left(\delta \rho_{\alpha}\right)=0$ and $\left.\left\langle\phi\left|\delta \rho_{\alpha}\right| \phi\right\rangle>0\right)$. Below we give all expressions to the first order in $\delta \hat{\Gamma}$ and $\delta \rho_{\alpha}$.

Expanding the tensor product inside the trace in Eq. (6) we get

$$
\begin{aligned}
& \prod_{\alpha=1}^{N} \otimes\left[\hat{\Gamma} \rho_{\alpha}\right]=(|\phi\rangle\langle\phi|)^{\otimes N} \\
& -\sum_{\alpha=1}^{N}(|\phi\rangle\langle\phi|)^{\otimes(\alpha-1)} \otimes R_{\alpha} \otimes(|\phi\rangle\langle\phi|)^{\otimes(N-\alpha)}
\end{aligned}
$$

with

$$
R_{\alpha}=\delta \rho_{\alpha}+\delta \hat{\Gamma}|\phi\rangle\langle\phi| .
$$

Let us denote by $C_{1}(\sigma)$ the set of fixed points (1-cycles) of a permutation $\sigma$. Then, computing the trace in Eq. 
(6) for the expression in Eq. (31) we obtain

$$
\begin{aligned}
J_{\sigma_{1}, \sigma_{2}} & =1-\sum_{\alpha \in C_{1}\left(\sigma_{2} \sigma_{1}^{-1}\right)} \operatorname{Tr}\left(R_{\alpha}\right)-\sum_{\alpha \notin C_{1}\left(\sigma_{2} \sigma_{1}^{-1}\right)}\left\langle\phi\left|R_{\alpha}\right| \phi\right\rangle \\
& =1-\sum_{\alpha=1}^{N}\langle\phi|\delta \hat{\Gamma}| \phi\rangle-\sum_{\alpha \notin C_{1}\left(\sigma_{2} \sigma_{1}^{-1}\right)}\left(1-\mathcal{F}_{\alpha}\right), \quad(33)
\end{aligned}
$$

where we have introduced the fidelity $\mathcal{F}_{\alpha}$ of a mixed internal state $\rho_{\alpha}$ as follows $\mathcal{F}_{\alpha}=\left\langle\phi\left|\rho_{\alpha}\right| \phi\right\rangle$. Since the trace of the third term on the r.h.s. of Eq. (33) is zero (all $\alpha$ are fixed points for $\sigma_{2}=\sigma_{1}$ ), using Eq. (10) we obtain

$$
p_{d}=1-\sum_{\alpha=1}^{N}\langle\phi|\delta \hat{\Gamma}| \phi\rangle .
$$

Therefore, from the definition $\mathcal{J}=J /\left(p_{d} N\right.$ !) we have

$$
\mathcal{J}_{\sigma_{1}, \sigma_{2}}=\frac{1}{N !}\left[1-\frac{1}{p_{d}} \sum_{\alpha \notin C_{1}\left(\sigma_{2} \sigma_{1}^{-1}\right)}\left(1-\mathcal{F}_{\alpha}\right)\right] \text {. }
$$

From Eqs. (19) and (35) we obtain

$$
1-p_{s}=\frac{1}{p_{d} N !} \sum_{\sigma} \sum_{\alpha \notin C_{1}(\sigma)}\left(1-\mathcal{F}_{\alpha}\right) .
$$

Let us introduce the minimal fidelity $\mathcal{F}=$ $\min \left(\mathcal{F}_{1}, \ldots, \mathcal{F}_{N}\right)$. Then the bound on the trace distance Eq. (12) can be estimated as follows

$$
1-p_{s} \leq \frac{1-\mathcal{F}}{p_{d} N !} \sum_{\sigma}\left[N-\left|C_{1}(\sigma)\right|\right]=\frac{N-1}{p_{d}}(1-\mathcal{F}),
$$

where $\left|C_{1}(\sigma)\right|$ is the total number of 1-cycles and we have used the well-known fact that $\frac{1}{N !} \sum_{\sigma}\left|C_{1}(\sigma)\right|=1$ [35].

It is seen from Eq. (37) that a realistic device with $N$ bosons is at a constant trace distance to the ideal bosonic network for the following scaling behavior of the minimal fidelity $\mathcal{F}=1-O(1 / N)$. The scaling law derived here is weaker by $\sqrt{N}$ then that obtained previously by a different method in Ref. [18]. In the next section we argue that the upper bound of Eq. (12), resulting in the above scaling law, is tight.

\section{LOWER BOUND ON THE TRACE DISTANCE AND DISTINGUISHABILITY CONJECTURE}

In section $\llbracket \mathrm{A}$ we have already mentioned the problem of uniqueness of $\mathcal{J}$-matrix for a given class of output probability distributions. Whereas in all of our calculations above such a $\mathcal{J}$-matrix was used as just an auxiliary concept whose uniqueness property was never used, now we have come to a point when the uniqueness property of $\mathcal{J}$-matrix plays an essential role (though in a formulation not as general as in section $\amalg$ A .
By Eq. (20) the difference between postselected output probabilities can be cast as

$$
p^{(i d)}(\vec{m})-p(\vec{m})=\left(1-p_{s}\right)\left[p^{(i d)}(\vec{m})-p^{(\perp)}(\vec{m})\right],
$$

where $p^{(\perp)}(\vec{m})$ corresponds to a density matrix $\mathcal{J}^{(\perp)}$ orthogonal to $\mathcal{J}^{(i d)}=|s\rangle\langle s|$ of the ideal bosonic input. Note that such a $\mathcal{J}^{(\perp)}$-matrix results in a nonclassical probability distribution at a network output. In particular, $\mathcal{J}_{\sigma_{1}, \sigma_{2}}^{(\perp)}=\operatorname{sgn}\left(\sigma_{2} \sigma_{1}^{-1}\right) / N$ ! describes completely indistinguishable bosons in an entangled (antisymmetric) internal state, simulating the output probability distribution of completely indistinguishable fermions, due to the boson-fermion duality [30].

Now, let us consider the maximum (taken over all $M \times M$-dimensional networks $U$ ) of the trace distance between the output probability distribution of an input resulting in a density matrix $\mathcal{J}^{(\perp)}$ and that of the ideal bosonic network

$$
d=\max _{\forall U} D\left(\mathbf{p}^{(i d)}, \mathbf{p}^{(\perp)}\right) .
$$

Then, by Eq. (38) the maximal over all $M \times M$ dimensional networks trace distance becomes (with the dependence on parameters given explicitly)

$$
\max _{\forall U} D\left(\mathbf{p}^{(i d)}, \mathbf{p}\right)=d\left(\mathcal{J}^{(\perp)}, N, M\right)\left(1-p_{s}(\mathcal{J}, N)\right) .
$$

At the next step the physical distinguishability between two mutually orthogonal $\mathcal{J}$-matrices plays an essential role. Let us state this property as the following.

Special distinguishability conjecture. - There is a constant $d_{0}>0$ bounding the function $d\left(\mathcal{J}^{(\perp)}, N, M\right)$ of Eq. (39) uniformly away from zero, i.e., $d\left(\mathcal{J}^{\left(\perp^{\prime}\right)}, N, M\right) \geq d_{0}$ for all $M, N \geq 2$ and all (physically realizable) $\mathcal{J}^{(\perp)}$ such that $\mathcal{J}^{(\perp)}|s\rangle=0$.

In other words, we conjecture that for any $N, M \geq 2$ one can always distinguish bosons in an internal state $\rho^{(i n t)}$ such that $S_{N} \rho^{(i n t)} S_{N}=0$ from the ideal bosonic case $S_{N} \rho^{(i n t)} S_{N}=\rho^{(i n t)}$ (with output probability distribution of Eq. (9) ) by comparing the respective output probability distributions over all unitary networks $U$.

If the above conjecture is true, then the upper bound of Eq. (12) is tight, i.e., it captures the correct scaling of the trace distance with $N$ (for instance, the scaling of the mutual fidelity for small errors derived in section III is then also tight). E.g., if we take $N=2$ the lower bound $\min \left(d\left(\mathcal{J}^{(\perp)}, 2, M\right)\right)$ is positive, since $\mathcal{J}^{(\perp)}$ is pure and corresponds to the ideal fermionic case (see also Ref. [36]).

From a physical point of view, at least a restricted version of the above conjecture (to some subset of all possible $\mathcal{J}^{(\perp)}$-matrices), relevant for a realistic BS device, must be true. Indeed, it is rather clear that if bosons have an internal state which is not a symmetric one, but a perturbation of a symmetric state, then the corresponding output distribution must have a nonzero maximum distance to the ideal bosonic network. 


\section{CONCLUSION}

We have employed a recently developed approach to partially indistinguishable bosons in a multi-mode unitary linear network for derivation of the upper and lower bounds on the trace distance of the respective output probability distribution to that of the ideal bosonic network. An important property was used, that in an auxiliary linear space, where the partial distinguishability matrix of bosons is defined, the boson counting measurement at a network output is formally represented also as a measurement. This fact allowed us to derive the upper and lower bounds on the trace distance by application of the standard methods. The form of the upper bound has a very clear physical interpretation: it is equal to the complementary of the probability of a symmetric input state of bosons, i.e., a state resulting in the ideal bosonic network.

In the case of a small distinguishability error there is a scaling law of the mismatch of internal states of bosons, stating that a realistic device with $N$ bosons is at a constant trace distance to the ideal bosonic network, if the minimal fidelity of internal states of bosons satisfies $\mathcal{F}=1-O(1 / N)$. This scaling law surpasses that obtained previously by a different method in Ref. [18].

The derived upper bound is tight (i.e., the lower bound has the same scaling in the number of particles as the upper one) provided that a physically clear distinguishability conjecture is true. The conjecture generalizes and puts in a mathematical form the universally assumed assumption that a perturbation of an ideal input state of bosons generally results in a non-ideal bosonic behavior. This touches upon the very important issue of the computational complexity of the BS. Indeed, if the distinguishability conjecture is false, then there are such non-ideal input states of only partially indistinguishable bosons, which have the same output probability distribution as the ideal BS with the completely indistinguishable bosons (since the trace distance between the two distributions would be zero).

Our main result can be also states as follows. We have given a tight experimentally accessible measure of "bosonness" of an unitary linear network with only partially indistinguishable bosons. The measure is given by the trace of projection of the internal state of bosons on the symmetric subspace of internal states, i.e., by the probability of having an input state symmetric in the internal degrees of freedom (which is also proportional to the probability of the maximally bunched output, which can be estimated from experimental data).

\section{ACKNOWLEDGEMENTS}

This work was supported by the CNPq (Brazil).

\section{Appendix A: Details of the proof of Theorem 1}

Let us first verify the following property of vectors $\left|Z_{\vec{l}}\right\rangle$

$$
\sum_{\vec{l}}\left|Z_{\vec{l}}\right\rangle\left\langle Z_{\vec{l}}\right|=\mathcal{P}, \quad \mathcal{P}_{\sigma_{1}, \sigma_{2}}=\frac{1}{\mu(\vec{n})} \sum_{\pi \in \mathcal{S}_{\vec{n}}} \delta_{\sigma_{2}, \pi \sigma_{1}}
$$

and show that $\mathcal{P}$ is a projector. Indeed, by unitarity of a network matrix $U$

$$
\begin{aligned}
& \sum_{\vec{l}}\left\langle\sigma_{1} \mid Z_{\vec{l}}\right\rangle\left\langle Z_{\vec{l}} \mid \sigma_{2}\right\rangle=\frac{1}{\mu(\vec{n})} \prod_{\alpha=1}^{N} \sum_{l_{\alpha}=1}^{M} U_{k_{\sigma_{1}(\alpha)}, l_{\alpha}} U_{k_{\sigma_{2}(\alpha)}, l_{\alpha}}^{*} \\
& =\frac{1}{\mu(\vec{n})} \prod_{\alpha=1}^{N} \delta_{k_{\sigma_{1}(\alpha)}, k_{\sigma_{2}(\alpha)}}=\frac{1}{\mu(\vec{n})} \sum_{\pi \in \mathcal{S}_{\vec{n}}} \delta_{\sigma_{2}, \pi \sigma_{1}} .
\end{aligned}
$$

Moreover, we have

$$
\mathcal{P}_{\sigma, \sigma^{\prime}}^{2}=\frac{1}{(\mu(\vec{n}))^{2}} \sum_{\sigma_{1}} \sum_{\pi_{1,2} \in \mathcal{S}_{\vec{n}}} \delta_{\sigma_{1}, \pi_{1} \sigma} \delta_{\sigma^{\prime}, \pi_{2} \sigma_{1}}=\mathcal{P}_{\sigma, \sigma^{\prime}}
$$

and $\operatorname{tr}(\mathcal{P})=\frac{N !}{\mu(\vec{n})}$.

\section{Appendix B: On the probability $p_{s}$}

We need to show that $p_{s}$ appears in the probability of a maximally bunched output configuration, say $\vec{l}_{B}=$ $(l, \ldots, l)\left(\right.$ i.e., $\left.m_{l}^{(B)}=N\right)$. Recalling that $\langle\sigma \mid s\rangle=1 / \sqrt{N !}$ we get (in this case there is just one measurement element for $\vec{m}_{l}^{(B)}$ )

$$
\left|Z_{\vec{l}_{B}}\right\rangle=\sqrt{\frac{N !}{\mu(\vec{n})}} \prod_{\alpha=1}^{N} U_{k_{\alpha}, l}|s\rangle
$$

Thanks to Eq. (B1), we have saturation of the upper bound (27) for an output probability of a maximally bunched output in the ideal bosonic case,

$$
p^{(i d)}\left(\vec{m}^{(B)}\right)=\frac{N !}{\mu(\vec{n})} \prod_{\alpha=1}^{N}\left|U_{k_{\alpha}, l}\right|^{2}=\frac{N !}{\mu(\vec{n})} p^{(c l)}\left(\vec{m}^{(B)}\right),
$$

whereas the other probability appearing in Eq. (38) vanishes, $p^{(\perp)}\left(\vec{m}^{(B)}\right)=0$. By resolving Eq. (38) we obtain the result

$$
p\left(\vec{m}^{(B)}\right)=p_{s} \frac{N !}{\mu(\vec{n})} p^{(c l)}\left(\vec{m}^{(B)}\right) .
$$


[1] S. Aaronson and A. Arkhipov, arXiv:1011.3245 [quantph]; Theory of Computing 9, 143 (2013).

[2] E. Knill, R. Laflamme, and G. J. Milburn, Nature 409 (2001) 46.

[3] S. D. Barlett and B. C. Sanders, J. Mod. Opt. 50, 2331 (2003).

[4] M. A. Nielsen and I. L. Chuang, Quantum Computation and Quantum Information, (Cambridge University Press, Cambridge, 2000).

[5] L. G. Valiant, Theoretical Coput. Sci., 8, 189 (1979).

[6] E. R. Caianiello, Nuovo Cimento, 10, 1634 (1953); Combinatorics and Renormalization in Quantum Field Theory, Frontiers in Physics, Lecture Note Series (W. A. Benjamin, Reading, MA, 1973).

[7] S. Aaronson, Proc. Roy. Soc. London A, 467, 3393 (2008).

[8] C. K. Hong, Z. Y. Ou, and L. Mandel, Phys. Rev. Lett. 59, 2044 (1987).

[9] Y. L. Lim and A. Beige, New J. Phys., 7, 155 (2005).

[10] M. A. Broome et al, Science 339, 794 (2013).

[11] J. B. Spring et al, Science, 339, 798 (2013).

[12] M. Tillmann et al, Nature Photonics, 7, 540 (2013).

[13] A. Crespi et al, Nature Photonics, 7, 545 (2013).

[14] N. Spagnolo et al, Phys. Rev. Lett. 111, 130503 (2013).

[15] P. P. Rohde and T. C. Ralph, Phys. Rev. A 85, 022332 (2012).

[16] P. P. Rohde, Phys. Rev. A 86, 052321 (2012).

[17] A. Leverrier and R. García-Patrón, arXiv:1309.4687 [quant-ph].

[18] V. S. Shchesnovich, Phys. Rev. A 89, 022333 (2014).

[19] V. S. Shchesnovich, arXiv:1403.4459 [quant-ph].

[20] C. Shen, Z. Zhang, and L.-M. Duan, Phys. Rev. Lett. 112, 050504 (2014).

[21] A. P. Lund, A. Laing, S. Rahimi-Keshari, T. Rudolph, J. L. O'Brien, and T. C. Ralph, Phys. Rev. Lett. 113, 100502 (2014).
[22] K. R. Motes, A. Gilchrist, J. P. Dowling, and P. P. Rohde, Phys. Rev. Lett. 113, 120501 (2014).

[23] S. Aaronson and A. Arkhipov, arXiv:1309.7460 [quant$\mathrm{ph}]$.

[24] N. Spagnolo et al, Nat. Photon. 8, 615 (2014).

[25] J. Carolan et al, Nat. Photon. 8, 621 (2014).

[26] M. C. Tichy, K. Mayer, A. Buchleitner, and K. Molmer, Phys. Rev. Lett. 113, 020502 (2014).

[27] J. Huh, G. G. Guerreschi, B. Peropadre, J. R. McClean, and A. Aspuru-Guzik, arXiv:1412.8427 [quant-ph].

[28] V. S. Shchesnovich, arXiv:1410.1506 [quant-ph].

[29] L. Mandel, Opt. Lett. 16, 1882 (1991).

[30] V. S. Shchesnovich, arXiv:1412.0279 [quant-ph]; to appear in Int. J. Quant. Inform. (2015).

[31] M. C. Tichy, Phys. Rev. A 91, 022316 (2015).

[32] J.-D. Urbina, J. Kuipers, Q. Hummel, and K. Richter, arXiv:1409.1558 [quant-ph].

[33] V. S. Shchesnovich, Int. J. Quantum Inform., 11, 1350045 (2013).

[34] K. Mayer, M. C. Tichy, F. Mintert, T. Konrad, and A. Buchleitner, Phys. Rev. A 83, 062307 (2011).

[35] R. P. Stanley, Enumerative Combinatorics, 2nd ed., Vol. 1 (Cambridge University Press, 2011).

[36] F. Töppel and A. Aiello, Phys. Rev. A 88, 012130 (2013).

[37] This problem is not trivial. The quadratic form of Eq. (5) is evaluated on mutually dependent variables $Z_{\sigma}=$ $\prod_{\alpha=1}^{N} U_{k_{\sigma(\alpha)}, l_{\alpha}}$, which fact could indicate that a given output probability distribution does not define a unique $J$-matrix. However, not every positive Hermitian matrix is a $J$-matrix, since it must be given by Eq. (6), possibly resulting in uniqueness of $J$-matrix.

[38] Eq. (21) follows from the general property [4] $\operatorname{tr}\{|\rho-\sigma|\}=\operatorname{tr}\left\{(\rho-\sigma)_{+}\right\}$where $(\rho-\sigma)_{+}$is the positive part of the difference between two density matrices, in our case $\left(1-p_{s}\right)|s\rangle\langle s|$. 